\title{
Influencers deportivos y su repercusión en el consumo, la actividad física y su proyección en redes sociales por los adolescentes andaluces (España) Sports influencers and their impact on consumption, physical activity and their projection on social networks by Andalusian adolescents (Spain)
}

Javier Gil Quintana, Rafael Felipe Ruiz, Miguel Ángel Moreno Muro

Universidad Nacional de Educación a Distancia (España)

Resumen. El objetivo de la presente investigación, cuyo corte metodológico es mixto, ha sido conocer el impacto que ejercen los influencers deportivos en un grupo poblacional de 404 adolescentes andaluces encuestados y 10 entrevistados cuya edad abarca desde los 16 hasta los 20 años. Atendiendo a los principales hallazgos, se demuestra que estos influencers contribuyen de manera positiva a aumentar el grado de motivación y adherencia por la actividad física. De forma sintética, el estudio establece una disociación entre la modalidad deportiva que se practica y el género del adolescente. Con esta afirmación queda expresado que ambos géneros tienen tendencia a seguir a influencers de su mismo género. Además, no se ha podido determinar que los hombres compren más dispositivos electrónicos que las mujeres. Sin embargo, un aspecto significativo a nivel estadístico es que el género masculino presenta más tendencia por compartir los datos en redes sociales y que los adolescentes que han comprado un dispositivo han intentado, en la mayor medida posible, que la marca se asemeje al que promociona su influencer. Concluimos que la labor que ejercen estos personajes públicos sobre sus seguidores es eficaz y directa, pues son capaces de incidir significativamente y mejorar la calidad de vida de todo aquel que los secunden.

Palabras clave. Influencers. Dispositivos digitales. Actividad deportiva. Fitness.

\begin{abstract}
The main of this research, whose methodological approach is mixed, has been to determine (otra opción: was to determine) the impact of sports influencers have on a population group of 404 Andalusian adolescents surveyed and 10 interviewees whose age ranges from 16 to 20 years. According to the main findings, it is shown that these influencers contribute positively to increasing the degree of motivation and adherence to physical activity. In summary, the study establishes a dissociation between the type of sport practiced and the gender of the adolescent. This statement shows that both genders tend to follow influencers of the same gender. In addition, it could not be determined that men buy more electronic devices than women. However, a statistically significant aspect is that males are more likely to share data on social media and that adolescents who have bought a device have tried, as much as possible, to have the brand resemble the one promoted by their influencer. Finally, it is concluded that the work of these public figures over their followers is effective and direct, as they are able to have a significant impact and improve the quality of life of all those who support them.
\end{abstract}

Keywords. Influencers. Digital wereables. Physical Activity. Fitness.

\section{Introducción}

Estamos inmersos en una sociedad Postdigital (Escaño, 2019). Con el desarrollo de las redes sociales (Instagram, Twitter, Facebook y TikTok), se han creado paulatinamente espacios más específicos en los que se puede interactuar con cualquier persona, crear grupos, enviar mensajes producir y consumir contenidos. Esta realidad es experimentada de forma específica por los niños, adolescentes y jóvenes que viven inmersos en estos espacios hiperconectados. El hecho de vivir como interactuantes e interactuados, ocasiona un movimiento constante y cotidiano a nivel digital, pero, en ocasio-

Fecha recepción: 17-05-20. Fecha de aceptación: 03-08-21

Javier Gil Quintana

jgilquintana@edu.uned.es nes, se convierte en un problema a nivel de salud, repercutiendo negativamente al desarrollar una vida sedentaria. Esta preocupación es latente desde el ámbito de la sanidad española, incluso se estima que va a aumentar en un 36\% la población sedentaria como señalaba la encuesta europea del 2014 (Encuesta Nacional de Salud, 2014), año en el que redes sociales como Instagram llevaban ya 4 años de proyección social, va a crecer hasta un 38,2\% al final del año 2025 según el informe anual 2018 La Sociedad en Red, transformación digital en España (Ministerio de Economía y Empresa, 2019).

Las tecnologías digitales se hacen presentes a nivel social y de consumo con el fin de crear tendencia hacia la práctica de la Actividad Física (AF) y se presentan dispositivos que, además de ofrecer posibilidades para un mejor rendimiento deportivo, también posibilitan la grabación de sesiones que se comparten en redes so- 
ciales (García-Fernández, et al., 2015) como medio de configuración en estos espacios y participación ciudadana (Flores Rivera, et al, 2021). De hecho, estos productos tracking (gadgets que registran un conjunto de parámetros de actividad física), son usados por más de un 20\% de la población americana (Jensen, 2021). Ello permite realizar un mejor seguimiento y/o control de la rutina deportiva. Son una excelente herramienta para aquellas personas que buscan mejorar y completar sus entrenamientos al permitir visualizar, controlar e interpretar los datos generados de su actividad (Veiga, 2017). No hay duda que, estos dispositivos digitales cada día cobran más relevancia en los centros deportivos como los gimnasios y, desde hace unos años, aparecen de forma sigilosa en algunos centros educativos respaldando prácticas del profesorado innovador (Moreno-Muciño, et al., 2021).

Puede resultar contraproducente que las tecnologías digitales y las redes sociales nos puedan mantener activos a nivel cognitivo, pero debemos preguntarnos ¿es posible que nos mantengan activos físicamente? El movimiento influencer puede darnos la respuesta, estas personas que tienen gran poder de influencia en los espacios sociales, con un gran número de followers y con un gran prestigio entre niños, adolescentes y jóvenes. A través de la motivación extrínseca la figura de los influencers (Greco, et al., 2015) se ha manifestado una respuesta en la etapa de la adolescencia presentando un mayor interés, entre otras cuestiones, por AF. El aspecto clave del buen influencer y el que más presente tienen las marcas, es su engagement que potencian con su target. Dicho de otra manera, la capacidad de un producto o una marca de establecer relaciones sólidas y duraderas con sus followers creando un compromiso que se establece entre la marca y los consumidores (Martínez Lemos, et al., 2016), entre AF y su práctica, entre AF y los hábitos de consumo que desarrolla.

La importancia de la investigación de corte metodológico mixto que presentamos a continuación queda justificada por la necesidad de descubrir el grado de motivación y adherencia hacia AF que ejercen los influencers sobre la etapa de la adolescencia tardía. Los influencers pueden convertirse en influencers de aprendizaje en el ámbito de la educación para la salud (GilQuintana, Malvasi, Castillo-Abdul, \& RomeroRodríguez, 2020), su labor debe ser talante de responsabilidad al influenciar con eficacia sobre sus followers, incidiendo significativamente y mejorando la calidad de vida de sus interactuados.

En base a estos planteamientos, presentamos este estudio que tiene como objetivo general descubrir el grado de motivación hacia el ejercicio físico que potencian los influencers deportivos, el género al que se asocia esta modalidad y la repercusión en el consumo de dispositivos y la proyección en redes sociales.

\section{Material y método}

\section{Método}

Esta investigación se ha realizado desde un enfoque mixto, una oportunidad de aportar riqueza al estudio porque, para descubrir la realidad social, se precisa de un enfoque polifacético y múltiple (Corbetta, 2007). Es por ello, que este estudio se desarrolla desde el paradigma positivista e interpretativo. La perspectiva positivista recoge aquellas investigaciones cuyo objeto de estudio sea el de verificar hipótesis a través de medios estadísticos o que tengan que establecer los ajustes paramétricos de una variable usando la expresión numérica. Desde el paradigma interpretativo ponemos el centro de atención en los aspectos no medibles u observables con métodos cuantitativos. Estos hechos no se miden, se interpretan y se evalúan de acorde a la realidad de la muestra (Ricoy, 2006). De acuerdo con esta propuesta se presentan a continuación los objetivos e hipótesis del estudio:

- Objetivo 1 (O1): Descubrir el grado de motivación por el ejercicio físico que ejercen los influencers deportivos.

- Objetivo 2 (O2): Descubrir qué género se asocia con cada modalidad deportiva.

- Objetivo 3 (O3): Comprobar si el género es un factor influyente a la hora de seguir influencers del mismo género u opuesto.

- Objetivo 4 (O4): Determinar si la población ha adquirido un dispositivo fitness $\mathrm{Y}$ detectar si los influencers repercuten en los adolescentes a la hora de realizar su compra.

- Objetivo 5 (O5): Apreciar el nivel de fijación por compartir una actividad deportiva en las redes sociales.

Estos objetivos se tienen presente, junto con las hipótesis siguientes:

- Hipótesis $1\left(\mathrm{H}_{1}\right)$ : La edad del adolescente es determinante a la hora de tener un influencer como referente.

- Hipótesis $2\left(\mathrm{H}_{2}\right)$ : La modalidad deportiva practicada por los adolescentes es importante a la hora de seguir un influencer.

- Hipótesis $3\left(\mathrm{H}_{3}\right)$ : El género del adolescente influye en el momento de seguir a un influencer. 
- Hipótesis $4\left(\mathrm{H}_{4}\right)$ : La práctica de actividad física de forma asidua, influye a la hora de tener influencer de referencia.

- Hipótesis $5\left(\mathrm{H}_{5}\right)$ : El uso de dispositivos digitales deportivo está ligado a la realización de actividad física de forma constante.

- Hipótesis $6\left(\mathrm{H}_{6}\right)$ : La modalidad deportiva practicada tiene relación con la adquisición de dispositivos electrónicos para realizar actividad física

- Hipótesis $7\left(\mathrm{H}_{7}\right)$ : El género y la edad son influyentes en los adolescentes a la hora de proyectar su actividad física en las redes sociales.

\section{Diseño}

La pregunta de investigación de la que parte nuestro estudio se engloba en qué repercusión tienen los influencers deportivos y cómo repercute en el consumo, la actividad física y la proyección en redes sociales en los adolescentes andaluces (España). Partiendo de esta cuestión, se desarrolló el plan de investigación que parte de una fundamentación teórica de los aspectos que se van a trabajar, seguido de la formulación de objetivos e hipótesis que sean comprobadas a través de técnicas de estudio cualitativo y cuantitativo, como son los cuestionarios y las entrevistas. La selección de la muestra respondía claramente al público que se quería estudiar: adolescentes andaluces. Y para ello, se desarrolló todo el proceso a lo largo de seis meses, en los que se efectuó el diseño de los instrumentos y su validación, la selección de la muestra y la recopilación de los datos. Finalmente, en base al análisis efectuado, se sacaron las conclusiones que, posteriormente serían compartidas con la comunidad científica.

\section{Procedimiento}

El proceso de estudio se realizó partiendo de un cuestionario digital on line difundido en entornos formativos formales y no formales y a través de las redes sociales. En el instrumento, las personas participantes podían leer el objeto de estudio y también las normas de utilización de los datos recolectados, consintiendo la recopilación de los mismos. Las entrevistas fueron obtenidas en base a la selección de 10 personas entre 16 y 21 años, quienes también pudieron leer el objeto de estudio y consentir la obtención de los datos cualitativos para nuestro estudio.

\section{Muestra}

La población objeto de estudio supone un total de 404 personas. De las personas participantes que res- pondieron el cuestionario, 218 tienen 16 años (54\% del total), 17 años (7,7\%), 21 años (5,2\%), 19 años (5\%) y 114 personas tienen 20 años (28,2\%). De las 404 personas encuestadas, son de género masculino el 45,8\% y de femenino el 54,2\% y la mayoría reside en núcleo urbano $(\mathrm{N}=257 / 63.6 \%)$. El nivel socioeconómico predominante es en su mayoría medio ( $\mathrm{N}=266.65,8 \%)$, siendo menor el nivel bajo $(\mathrm{N}=63.15,6 \%)$ y el alto $(\mathrm{N}=$ 75. 18.6\%). Dentro de la formación académica, los sujetos escolarizados en Educación Secundaria Obligatoria representan el 36,9\% ( $=149)$, en Bachillerato el 30,2\% ( $N=122)$, en Formación Profesional el 25,5\% $(\mathrm{N}=30)$ y el porcentaje con estudios universitarios es el 25,5\% ( $\mathrm{N}=103)$. Referido a la muestra del estudio cualitativo, se realizó a un grupo de jóvenes de edades comprendidas entre los 16 y 21 años, 10 adolescentes, $60 \%$ de género masculino y $40 \%$ femenino.

\section{Instrumento}

Esta investigación se apoya tanto en la técnica cuantitativa utilizando el cuestionario como en la herramienta cualitativa de las entrevistas semiestructuradas. El cuestionario es el instrumento más utilizado con la finalidad de obtener datos en estudios de sociología empírica (García et al., 2015); en nuestro caso, consta de 30 ítems dividido en tres categorías sobre consumo de influencers deportivos, consumo de publicidad deportiva, y actividad deportiva proyectada en redes sociales. Se recogen 12 ítems; 2 son medidos sobre sí/no y el resto mediante escala Likert, para conocer el grado de «desacuerdo» o «de acuerdo» en relación con cuestiones vinculadas con la actitud. El resto de ítems (del 13 al 30), evalúan aspectos relacionados con las 3 categorías anteriormente mencionadas. La entrevista está formada por planteamiento individual semiestructurado en torno a 10 preguntas que fueron realizadas a 5 hombres y 5 mujeres seleccionadas de personas que realizan deporte de forma profesional, como aficionadas y también relacionadas con el ámbito educativo y comunicativo. Los sujetos fueron debidamente informados de que formaban parte de un estudio con unos objetivos e hipótesis concretas, aceptando el consentimiento de forma firmada y garantizando su anonimato a nivel de datos. Tanto el cuestionario como las entrevistas fueron organizadas en todo a ámbitos que responden a las categorías en base a la estructura realizada en el análisis de resultados.

\section{Análisis estadístico}

Para hacer el análisis cuantitativo de los datos se ha 
empleado el software estadístico IBM SPSS. Los datos recogidos en la entrevista, conversación guiada por el equipo investigador que se realiza a los sujetos seleccionados en la muestra a través de preguntas sin estandarizar y flexibles (Corbetta, 2007), se han analizado con el software Atlas.ti 9.0, con la intención de tener una conclusión consensuada del instrumento utilizado. Los códigos pueden o no pueden ser vinculados a citas textuales; espacios para la integración, la reflexión, el análisis, y la interpretación.

\section{Validez de los instrumentos y fiabilidad de los datos}

La validad de los instrumentos ha seguido un juicio de cinco exper tos, como comité de ética, concretamente personas del grupo de investigación SMEMIU (UNED). Para las pruebas de independencia, que nos permiten ver la posible asociación entre nuestras variables, se aplicó test chi-cuadrado y test de Fisher, pues todas nuestras variables son cualitativas (categóricas), detallándose los resultados en las distintas tablas. En relación con la consistencia interna del instrumento, se recurrió a test Alfa de Cronbach, el cual permite estimar la fiabilidad de un instrumento. Esta medida de la fiabilidad asume que los ítems (medidos en escala tipo Likert o dicotómicas) miden un mismo constructo y están altamente correlacionados. Cuanto más cerca se encuentre el valor del alfa a 1 mayor es la consistencia interna de los ítems analizados. Por último, el nivel de significación ( $\mathrm{p}>$ valor), se estableció en 0,706 (Tabla 1).

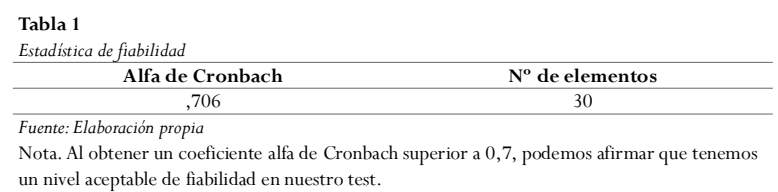

\section{Resultados}

\section{tivos \\ Categoría I. Perfil followers influencers depor-}

En relación con el perfil de followers, encontramos diferencias significativas en función de dos variables: edad y género. Existen diferencias significativas entre la correlación tener influencer y edad; de las 218 personas que tienen 16 años, el 61\% sí tiene influencer; el 29\% de personas de 17 sí que tienen influencers; a los 18 años el 57,1\%, con 19 un 65\% y los que tienen 20 años un $61,4 \%$. Este se respalda gracias al p-valor de chi cuadrado (p valor: 0,014$)$, que sale significativo $(p<0,05)$. Por tanto, con un nivel de confianza del 95\% podemos aceptar la hipótesis de dependencia entre las variables.
La segunda variable es el género, presentada en la Tabla 4. De los 185 hombres encuestados, el 58,9\% ( $N=109$ personas) sí sigue a influencers deportivos, el 41,1\% no $(\mathrm{N}=76)$. En el género femenino de las 219 , el 58,4\% $(\mathrm{N}=128)$ siguen influencers deportivos mientras que el 41,6\% ( $\mathrm{N}=91)$ niega seguirlos. El p-valor de la prueba de independencia chi cuadrado ( $p$ valor: 0,0924) sale no significativo ( $>0,05)$, por tanto, podemos aceptar la hipótesis de independencia entre las variables y comprobar los valores correlacionales en la Tabla 2 y 3 . Tomando como valores referentes los ofrecidos por test de Spearman como consecuencia de que nuestra muestra sigue una distribución no paramétrica (no normal), tomamos todos los valores que oscilen entre -1 (correlación débil) y 1 (correlación fuerte). Observamos que la correlación entre estas variables es fuerte, ya que el valor obtenido (,825), se aproxima bastante a 1 (Tabla $2)$. En este caso, existe una relación proporcional entre seguir influencer y la edad de la población encuestada. La correlación entre las variables tener influencers y género es positiva y, además, fuerte, ya que el valor obtenido (,924) se aproxima a 1 (Tabla 3). Descubrimos que el género no es determinante para que sigan a influencers.

Tabla 2

\begin{tabular}{|c|c|c|c|c|}
\hline & Valor & $\begin{array}{l}\text { Error estándar } \\
\text { asintótico }\end{array}$ & $\mathrm{T}$ aproximada ${ }^{\mathrm{b}}$ & $\begin{array}{l}\text { Significación } \\
\text { aproximada }\end{array}$ \\
\hline Intervalo por intervalo R de Pearson &,- 018 & ,049 &,- 369 &, $712^{\mathrm{c}}$ \\
\hline Ordinal por ordinal $\begin{array}{l}\text { Correlación de } \\
\text { Spearman }\end{array}$ & ,011 & ,049 & ,221 &, $825^{c}$ \\
\hline $\mathrm{N}$ de casos válidos & 404 & & & \\
\hline \multicolumn{5}{|l|}{ Fuente. Elaboración propia } \\
\hline \multicolumn{5}{|l|}{ Tabla 3} \\
\hline & Valor & $\begin{array}{c}\text { Error estándar } \\
\text { asintótico }^{\mathrm{a}}\end{array}$ & T aproximada ${ }^{b}$ & $\begin{array}{r}\text { Significación } \\
\text { aproximada }\end{array}$ \\
\hline Intervalo por intervaloR de Pearson & 005 & ,050 & ,096 &, $924^{c}$ \\
\hline $\begin{array}{ll}\text { Ordinal por ordinal } & \begin{array}{l}\text { Correlación de } \\
\text { Spearman }\end{array}\end{array}$ & ,005 & ,050 & ,096 & , $924^{c}$ \\
\hline $\mathrm{N}$ de casos válidos & 404 & & & \\
\hline
\end{tabular}

Para conocer la conexión entre personas entrevistadas y los posibles influencers que marcan su vida e intervienen en el ámbito cotidiano de cada uno (Figura 1), se encuentra que la gran mayoría, independientemente del género, tienen como principales influencers a personas famosas que, posteriormente, confiesan que son ídolos. Cabe destacar que practican un entrenamiento más de fuerza que se puede realizar en el gimnasio, manifiestan que tratan de seguir a influencers que tienen como objetivo el entrenamiento y AF. Este dato que presenta el estudio cualitativo es manifestado por hombres entre 16 y 17 años, por lo que se puede empezar a ver la influencia en la etapa de la adolescencia al culto al cuerpo.

Continuando con el análisis cualitativo observamos 


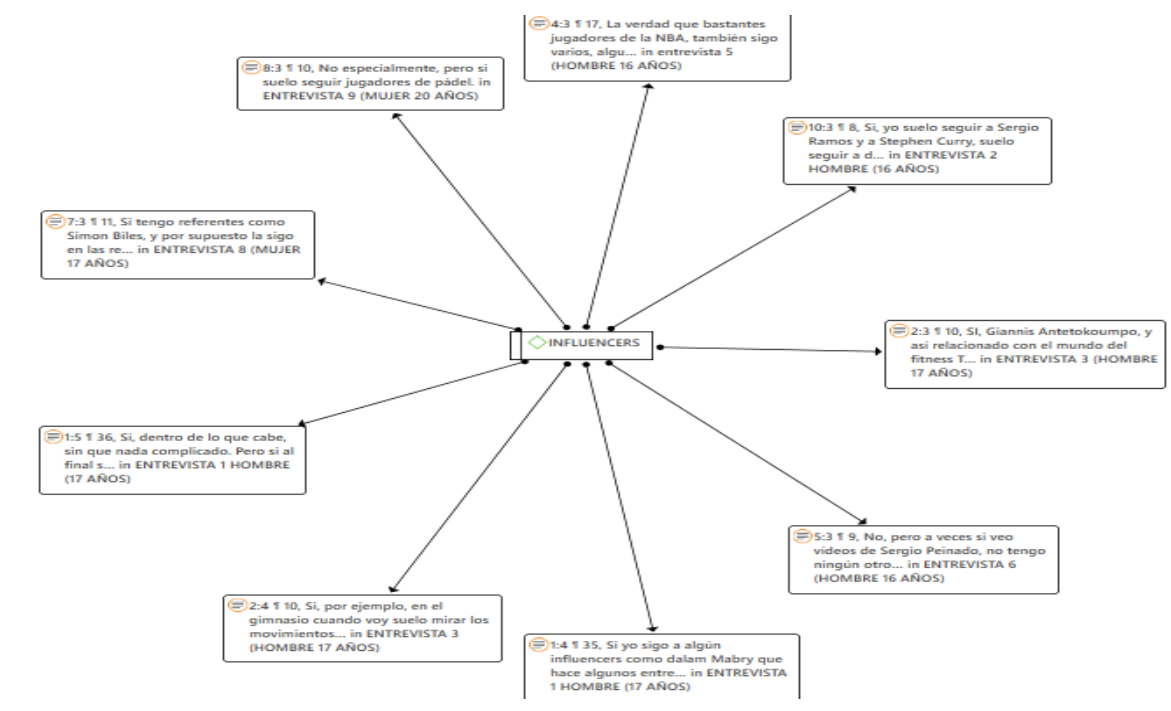

Figura 1

Referente en redes relacionado con AF, deportista famoso o influencer. Fuente: elaboración propia. los varones que tienen influencer femenino, el porcentaje solo asciende al $9,2 \%(\mathrm{~N}=12)$. Las mujeres el $71,7 \%$ se decantan más por influencer de igual género $(\mathrm{N}=104)$; prefieren un influencer hombre el 28,3\% ( $\mathrm{N}=41)$. El p-valor de la prueba de independencia chi cuadrado ( $\mathrm{p}$ valor: $0,000)$ sale significativo ( $\mathrm{p}<0,05)$, por tanto, con un nivel de confian- que dos de las personas se muestran indiferentes ante la asociación de tener influencer deportivo al que seguir en redes sociales, concretando que son mujeres de 16 y 18 años. Hay que destacar la entrevista 9, que la respuesta inicial a la pregunta es negativa, pero luego si aclara que sigue a jugadores de pádel. Este dato podemos asociar la respuesta que al ver el número de followers de influencers de pádel, esta entrevistada no lo asociaba a influencers como tal (Figura 2). za del 95\% podemos aceptar la hipótesis de dependencia, entre las variables.

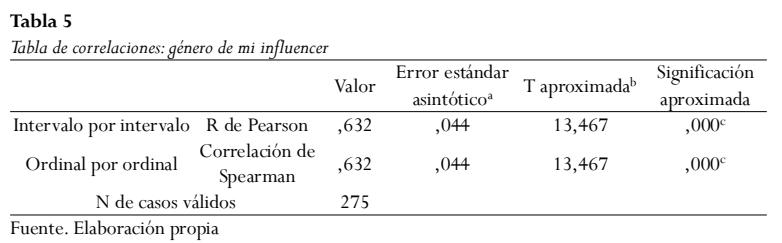

En la tabla 5 podemos consultar la correlación establecida de la anterior tabla cruzada. Observamos que la correlación entre estas variables es débil, pero positiva, ya que el valor obtenido $(, 000)$ queda lejos de a 1. Tanto hombres como mujeres prefieren seguir a influencers de su mismo género, aunque la correlación salga débil (Tabla 5).

\section{Figura 2}

Referente en redes relacionado con AF, deportista famoso o influencer. Fuente: Elaboración propia.
Para 52 personas que practican deporte, el 58,4\% afirma que los influencers no le han condicionado nada. Si seguimos la escala, el 63,9\% (N = 39) señalan que los influencers han influido poco en su afán por la AF. Ya en un nivel medio, el 69,3\% ( $\mathrm{N}=52)$ siente que sus hábitos se han visto mejorados gracias al influencer de forma «aceptable». Si nos centramos en las dos últimas categorías, el 91,4\% de los que realizan deporte dejan constancia de que el influencers interfiere «bastante». El 91,7\% ( $\mathrm{N}=66)$ afirma que sus hábitos se han visto mejorados por el influencer. Finalmente, en la categoría «mucho», el 91,4\% ( $\mathrm{N}=32)$ cree que han influido 
de forma positiva en su adherencia por la AF. Para esta correlación, el p-valor de la prueba de independencia chi cuadrado ( $p$ valor: 0,000$)$ sale significativo $(p<0,05)$, por tanto, con un nivel de confianza del $95 \%$ podemos aceptar la hipótesis de dependencia entre las variables (Tabla 6).

Tabla 6

\begin{tabular}{|c|c|c|c|c|c|}
\hline & & & \multicolumn{2}{|c|}{ Práctica $\mathrm{AF}$} & \multirow{2}{*}{ Total } \\
\hline & & & Sí & No & \\
\hline \multirow{12}{*}{$\mathrm{X} 1$} & \multirow{2}{*}{ Nada } & Recuento & 52 & 37 & 89 \\
\hline & & $\%$ Práctica AF & $58,4 \%$ & $41,6 \%$ & 100 \\
\hline & \multirow{2}{*}{ Poco } & Recuento & 39 & 22 & 61 \\
\hline & & $\%$ Práctica AF & $63,9 \%$ & $36,1 \%$ & $100 \%$ \\
\hline & \multirow{2}{*}{ Aceptable } & Recuento & 52 & 23 & 75 \\
\hline & & $\%$ Práctica $\mathrm{AF}$ & $69,3 \%$ & $30,7 \%$ & $100 \%$ \\
\hline & \multirow{2}{*}{ Bastante } & Recuento & 66 & 6 & 72 \\
\hline & & $\%$ Práctica AF & $91,7 \%$ & $8,3 \%$ & $100 \%$ \\
\hline & \multirow{2}{*}{ Mucho } & Recuento & 32 & 3 & 35 \\
\hline & & $\%$ Práctica AF & $91,4 \%$ & $8,6 \%$ & $100 \%$ \\
\hline & \multirow{2}{*}{ Total } & Recuento & 241 & 91 & 332 \\
\hline & & $\%$ Práctica AF & $100,0 \%$ & $100,0 \%$ & $100,0 \%$ \\
\hline
\end{tabular}

Observamos que la correlación entre estas variables (Tabla 7) es de intensidad media, ya que el valor obtenido (,558) está cerca de 1 . Esto significa que podemos afirmar que las personas que realizan deporte, en más de la mitad de los casos, van a consumir contenido de influencers deportivos.

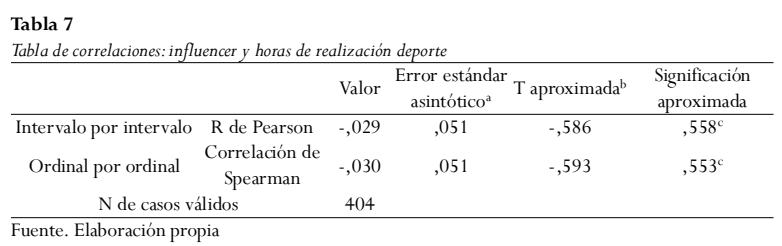

Se demuestra que las personas que más tiempo dedican a la práctica de AF a la semana (más de 6 horas), tienen más probabilidad de tener influencer deportivo un 27,0\%, ( $\mathrm{N}=64)$. Aquellos que dedican entre 4 a 6 horas, el 22,4\%, sigue a algún influencer, y el 15,0\% no. A medida que vamos reduciendo en número de horas, de aquellas personas que dedican de 2 a 4 horas, el 18,6\% es consumidor de influencers, mientras que el 24,6\% no (Tabla 8). En lo más bajo de la escala encontramos que el tiempo de AF semanal oscila de 1 a 2 horas. El $17,7 \%(\mathrm{~N}=42)$, sí tiene influencer deportivo, y el $22,2 \%$ no $(\mathrm{N}=37)$. Como el $\mathrm{p}$ valor $(0,002)$ de esta correlación sale significativo $(\mathrm{p}<0,05)$ podemos aceptar la hipótesis de dependencia entre las variables. Otro aspecto estudiado es el hecho de si el género determina una modalidad deportiva en concreto. El perfil deportivo para la mayoría de la población encuestada es el fitness (57,9\% hombres y 56,2\% mujeres). La segunda modalidad más repetida es el fútbol (24\% hombres y 21,7\% mujeres). En tercer lugar, aparece el running (11,5\% hombres, $\mathrm{N}=21$, y $12 \%$ mujeres $\mathrm{N}=26$ ). Las modalidades deportivas por las que se tiene menos interés son el ciclismo $(3,3 \%$ hombres, $\mathrm{N}=6$, y $6,5 \%$ muje- res, $\mathrm{N}=14)$, y el tenis (3,3\% hombres, $\mathrm{N}=6$, y 3,7\% mujeres, $\mathrm{N}=8)$. Estos datos, junto al p-valor de la prueba de independencia chi cuadrado $(0,669)$, sale no significativo ( $p>0,05)$. Por tanto, con un nivel de confianza del 95\% podemos aceptar la hipótesis de independencia entre las variables.

Tabla 8

Tabla cruzada: perfil influencer con género

\begin{tabular}{|c|c|c|c|c|c|}
\hline & & & \multicolumn{2}{|c|}{ Género } & \multirow{2}{*}{ Total } \\
\hline & & & Masculino & Femenino & \\
\hline \multirow{10}{*}{ Perfil de mi influencer } & \multirow{2}{*}{ Fitness } & Recuento \% & 106 & 122 & 228 \\
\hline & & & $57,9 \%$ & $56,2 \%$ & $57,0 \%$ \\
\hline & \multirow{2}{*}{ Ciclismo } & Recuento \% & 6 & 14 & 20 \\
\hline & & & $3,3 \%$ & $6,5 \%$ & $5,0 \%$ \\
\hline & \multirow{2}{*}{ Fútbol } & Recuento \% & 44 & 47 & 91 \\
\hline & & & $24,0 \%$ & $21,7 \%$ & $22,8 \%$ \\
\hline & \multirow{2}{*}{ Running } & Recuento \% & 21 & 26 & 47 \\
\hline & & & $11,5 \%$ & $12,0 \%$ & $11,8 \%$ \\
\hline & \multirow{2}{*}{ Tenis } & Recuento \% & 6 & 8 & 14 \\
\hline & & & $3,3 \%$ & $3,7 \%$ & $3,5 \%$ \\
\hline \multirow{2}{*}{\multicolumn{2}{|c|}{ Total }} & Recuento \% & 183 & 217 & 400 \\
\hline & & & $100,0 \%$ & $100,0 \%$ & $100,0 \%$ \\
\hline
\end{tabular}

Observamos que la correlación entre estas variables es positiva, ya que el valor obtenido $(, 010)$ es superior a 0 (Tabla 9). Existe una relación proporcional entre ser hombre o mujer y seguir cierta modalidad deportiva.

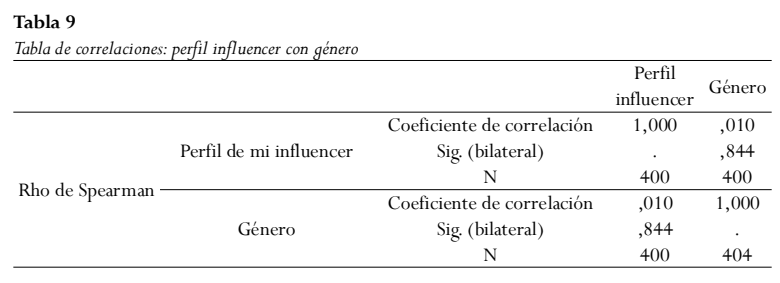

La población entrevistada corresponde a adolescentes, mujeres y hombres, que practican bastante AF semanalmente. Independientemente de género y edad, la relación existente entre la $\mathrm{AF}$ en un club deportivo y su adherencia es aparente entre la muestra entrevistada, ya que la media semanal sobrepasa las 4 horas. Observamos que superan considerablemente la media entre edades correspondientes a nivel estatal según detalla el Anuario de Estadísticas Deportivas (2019) del Ministerio español de Cultura y Deporte (Figura 3).

\section{Categoría II. Consumo de dispositivos digitales deportivos}

En relación con el consumo de dispositivos digitales el 60\% ( $\mathrm{N}=111)$ de los hombres afirman haber comprado un gadget deportivo, frente al 49,3\% $(\mathrm{N}=108)$ de mujeres. Entre quienes prescinden de adquirir este tipo de dispositivos, el 40\% ( $\mathrm{N}=74)$ son hombres, y el $50,7 \%$ mujeres $(\mathrm{N}=111)$. Como el $\mathrm{p}$ valor $(0,032) \mathrm{de}$ esta correlación sale significativo $(\mathrm{p}<0,05)$ podemos aceptar la hipótesis de dependencia entre las variables (Tabla 10). 


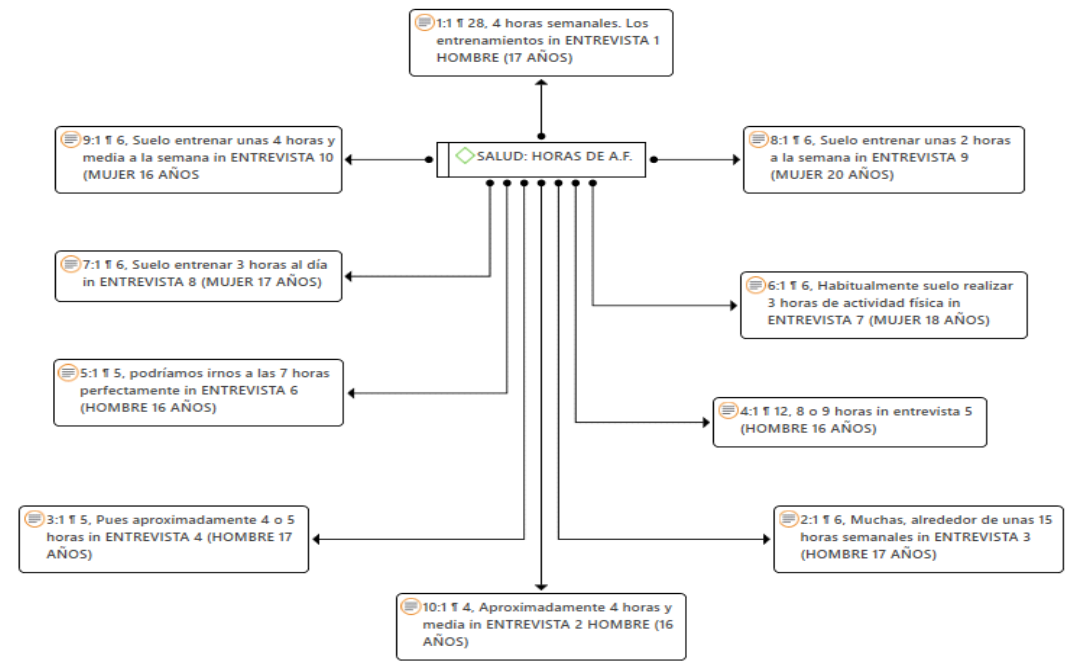

Figura 3

Horas $A F$ realizadas semanalmente. Fuente: elaboración propia.

Tabla 10

Tabla cruzada: adquisición de un dispositivo digital - género

\begin{tabular}{|c|c|c|c|c|c|}
\hline & & & & & \\
\hline & & & Masculino & Femenino & Total \\
\hline & & Recuento \% & 111 & 108 & 219 \\
\hline Adquisición de & 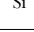 & & $60,0 \%$ & $49,3 \%$ & $54,2 \%$ \\
\hline un dispositivo & & Recuento \% & 74 & 111 & 185 \\
\hline & No & & $40,0 \%$ & $50,7 \%$ & $45,8 \%$ \\
\hline & & Recuento \% & 185 & 219 & 404 \\
\hline & & & $100,0 \%$ & $100,0 \%$ & $100,0 \%$ \\
\hline
\end{tabular}

Observamos que la correlación entre estas variables es positiva, ya que el valor obtenido $(, 107)$ es superior a 1 . Este dato invita a pensar que los hombres tienen a comprar de forma proporcional, dispositivos digitales fitness, en detrimento de las mujeres, aunque no sea la correlación fuerte (Tabla 11).

Tabla 11

\begin{tabular}{|c|c|c|c|c|}
\hline & & & $\begin{array}{c}\text { Adquisición de } \\
\text { dispositivo digital }\end{array}$ & Género \\
\hline \multirow{6}{*}{$\begin{array}{l}\text { Rho de } \\
\text { Spearman }\end{array}$} & \multirow{3}{*}{$\begin{array}{l}\text { ¿He adquirido un dispositivo } \\
\text { electrónico deportivo? }\end{array}$} & Coeficiente de correlación & 1,000 & $107^{*}$ \\
\hline & & Sig. (bilateral) & & ,032 \\
\hline & & $\mathrm{N}$ & 404 & 404 \\
\hline & \multirow{3}{*}{ Sexo } & Coeficiente de correlación &, $107^{*}$ & 1,000 \\
\hline & & Sig. (bilateral) & ,032 & . \\
\hline & & $\mathrm{N}$ & 404 & 404 \\
\hline
\end{tabular}

En la muestra quienes practican deporte entre 1 y 2
El grupo que practica de 4 a 6 horas semanales, el $24,7 \%$ ha adquirido un gadget. En el extremo, las personas que dedican más de 6 horas semanales $(60,2 \%)$, el $24,2 \%$ tiene un dispositivo para monitorizar su AF. Aquí el p valor sale significativo $(0,000)$ (p $<0,05)$ Las personas que practican alguna $\mathrm{AF}$, el $78,1 \%(\mathrm{~N}=171)$, ha comprado dispositivo para monitorizar sus entrenamientos. Por otro lado, el 59,5\% manifiesta no haber realizado dicha adquisición. El p valor $(0,000)$ sale significativo $(\mathrm{p}<0,05)$. Observamos que la correlación entre estas variables es positiva, ya que el valor obtenido (,202) es superior a 1 (Tabla 12). Aumentar considerablemente el número de horas dedicadas a la práctica deportiva es directamente proporcional a la toma de decisión de adquirir un dispositivo fitness.

Tabla 12

Tabla de correlación: práctica de actividad deportiva y adquisición de dispositivo digital

\begin{tabular}{|c|c|c|c|c|}
\hline & \multirow{2}{*}{\multicolumn{2}{|c|}{$\begin{array}{c}¿ \text { Practicas } \\
\text { alge adquirido un } \\
\text { algua actividad dispositivo electrónico }\end{array}$}} \\
\hline & & & & \\
\hline & & & deportiva? & deportivo? \\
\hline \multirow{6}{*}{$\begin{array}{l}\text { Rho de } \\
\text { Spearman }\end{array}$} & \multirow{3}{*}{$\begin{array}{l}\text { ¿Practicas alguna } \\
\text { actividad deportiva? }\end{array}$} & Coeficiente de correlación & 1,000 &, $202^{* *}$ \\
\hline & & Sig. (bilateral) & &, 000 \\
\hline & & $\mathrm{N}$ & 404 & 404 \\
\hline & ¿He adquirido un & Coeficiente de correlación &, $202^{* *}$ & 1,000 \\
\hline & dispositivo electrónico & Sig. (bilateral) & ,000 & \\
\hline & deportivo? & $\mathrm{N}$ & 404 & 404 \\
\hline
\end{tabular}

**. La correlación es significativa en el nivel 0,01 (bilateral).

En el estudio cualitativo odemos encontrar respuestas negativas al uso de dispositivos para medir AF apreciando que el género no influye en el uso de dispositivos para medirla. La modalidad deportiva que practican sí es influyente en el uso de dispositivos entre la población horas semanales el $16,4 \%$ ha comprado un dispositivo digital, mientras el $23,2 \%$ no lo ha hecho. A medida que subimos las horas semanales practicando deporte, la posibilidad de comprar dispositivo aumenta. Los que destinan entre 2 a 4 horas, el $23,3 \%$ ha adquirido un wereable y el $18,4 \%$ no.

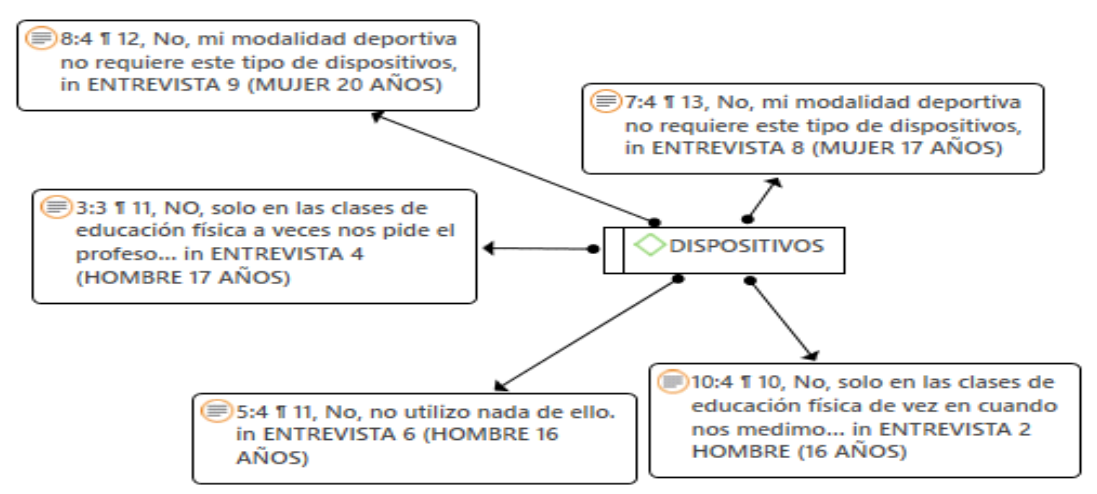

Figura 4

Realización de AF y uso de dispositivo digital. Fuente: elaboración propia. 
entrevistada; coinciden con quienes practican deportes de equipo o de contacto. Las personas que practican deportes individuales que requieren entrenamiento específico sí lo suelen utilizar (Figura 4).

Las personas entrevistadas que practican deportes individuales como observamos en la Figura 5, como son atletismo y ciclismo, usan este tipo de dispositivos, valorándolo positivamente. La muestra nos habla de la red social para compartir entrenamientos como es Strava, con influencia positiva; o el uso de smartwatch como forma de prevención hacia el sedentarismo.

Dando un paso más en el ámbito del consumo, ob- de los casos, la marca que promociona su influencer no es un aspecto relevante para ellos.

Tabla 14

Tabla de correlaciones: adquirir dispositivo - marca

\begin{tabular}{|c|c|c|c|c|c|}
\hline & & Valor & $\begin{array}{c}\text { Error estándar } \\
\text { asintótico }^{\mathrm{a}}\end{array}$ & $\mathrm{T}$ aproximada $^{\mathrm{b}}$ & $\begin{array}{l}\text { Significación } \\
\text { aproximada }\end{array}$ \\
\hline Intervalo por intervalo & $R$ de Pearson &,- 156 & ,050 & $-2,922$ & ,004c \\
\hline Ordinal por ordinal & $\begin{array}{l}\text { Correlación de } \\
\text { Spearman }\end{array}$ &,- 173 & ,052 & $-3,250$ &, $001^{c}$ \\
\hline \multicolumn{2}{|c|}{$\mathrm{N}$ de casos válidos } & 346 & & & \\
\hline
\end{tabular}

Entre el grupo de adolescentes que ha comprado un dispositivo digital, el 42,9\% $(\mathrm{N}=27)$ presenta un nivel socioeconómico bajo. El 55,6\% ( $\mathrm{N}=148)$ representa nivel medio y el 58,7\% ( $\mathrm{N}=44)$ se encuentra en nivel alto. Aquellas personas que no han adquirido wereables deportivos, el $57,1 \%(\mathrm{~N}=36)$ representa el nivel bajo, el $44,4 \%(\mathrm{~N}=118) \mathrm{de}-$ fiende el nivel medio y el nivel alto tiene una cota de 41,3\% $(\mathrm{N}=31)$. El p-valor de la prueba de independencia chi cuadrado $(0,129)$ sale no significativo $(p>0,05)$. Con un nivel de confian-

\section{Figura 5}

Uso de dispositivo para medir la AF. Fuente: elaboración propia.

servamos que las personas que han comprado un gadget tecnológico el 49,5\% ( $\mathrm{N}=107)$ ha adquirido un dispositivo sin importar que la marca o modelo fuese igual al de su influencer; y el 64,1\% ( $\mathrm{N}=25)$ se muestra indiferente (Tabla 13). En la escala de los que están de acuerdo con adquirir un producto semejante o parecido, contamos con el 61,1\%, de representación ( $\mathrm{N}=11)$. Aquellos que están totalmente de acuerdo, el 77,8\%, ha intentado que la marca del dispositivo fuese igual o parecido al del influencer. En este caso aceptamos la relación de dependencia al obtener un p valor $(0,000)$ inferior a ,005.

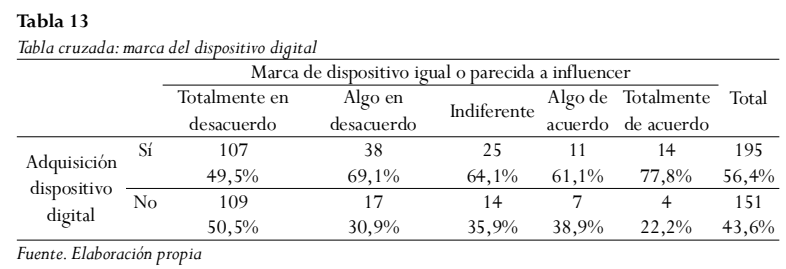

Observamos que la correlación entre estas variables es positiva y débil, ya que el valor obtenido $(, 004)$ es escasamente superior a 0 (Tabla 14). En base a los datos adquiridos y en función de las características que este grupo poblacional manifiesta, en más de la mitad za del 95\% podemos aceptar la hipótesis de independencia entre las variables.

\section{Categoría III. Proyección AF en redes sociales}

Entre los adolescentes que señalan estar totalmente de acuerdo con la importancia que otorgan a los datos post AF (X2), el 39\% comparte esos datos en Instagram al igual que sus influencers. Podemos apreciar cómo, a medida que esa importancia por los datos aumenta, el porcentaje que postea la AF también se incrementa, pues los que se posicionan en el bando de los que comparten los datos, el 7,8\% se siente «totalmente en desacuerdo», el 10,4\% afirma estar algo de acuerdo, y el $15,6 \%$ manifiesta encontrarse indiferente ante esta cuestión, como observamos en la Tabla 15.

\begin{tabular}{|c|c|c|c|c|c|}
\hline \multicolumn{6}{|c|}{ Compartir datos de mi } \\
\hline & & & \multicolumn{2}{|c|}{$\mathrm{AF}$ en redes sociales } & \multirow[t]{2}{*}{ Total } \\
\hline & & & Sí & No & \\
\hline \multirow{10}{*}{$\mathrm{X} 2$} & Totalmente en & Recuento $\%$ & 6 & 61 & 67 \\
\hline & desacuerdo & & $7,8 \%$ & $21,4 \%$ & $18,5 \%$ \\
\hline & \multirow{2}{*}{ Algo en desacuerdo } & Recuento \% & 8 & 48 & 56 \\
\hline & & & $10,4 \%$ & $16,8 \%$ & $15,5 \%$ \\
\hline & \multirow{2}{*}{ Indiferente } & Recuento \% & 12 & 64 & 76 \\
\hline & & & $15,6 \%$ & $22,5 \%$ & $21,0 \%$ \\
\hline & \multirow{2}{*}{ Algo de acuerdo } & Recuento \% & 21 & 57 & 78 \\
\hline & & & $27,3 \%$ & $20,0 \%$ & $21,5 \%$ \\
\hline & Totalmente de & Recuento \% & 30 & 55 & 85 \\
\hline & acuerdo & & $39,0 \%$ & $19,3 \%$ & $23,5 \%$ \\
\hline
\end{tabular}


Observamos que la correlación entre estas variables es negativa, ya que el valor obtenido (-034) es inferior al valor 0 (Tabla 16).

Tabla 16

Tabla de correlaciones: los datos conseguidos post AF y proyección en redes sociales

\begin{tabular}{|c|c|c|c|c|}
\hline & & & $\begin{array}{c}\text { Importancia datos } \\
\text { conseguidos } \\
\text { después de AF }\end{array}$ & $\begin{array}{c}\text { Compartir } \\
\text { datos en } \\
\text { redes sociales }\end{array}$ \\
\hline \multirow{6}{*}{$\begin{array}{l}\text { Rho de } \\
\text { Spearman }\end{array}$} & \multirow{3}{*}{$\begin{array}{l}\text { Importancia datos } \\
\text { conseguidos después de AF }\end{array}$} & Coeficiente de correlación & 1,000 &,$- 034^{* *}$ \\
\hline & & Sig. (bilateral) & & , 000 \\
\hline & & $\mathrm{N}$ & 364 & 362 \\
\hline & \multirow{3}{*}{$\begin{array}{l}\text { Compartir datos AF en } \\
\text { redes sociales. }\end{array}$} & Coeficiente de correlación &,$- 234^{* *}$ & 1,000 \\
\hline & & Sig. (bilateral) & , 000 & \\
\hline & & $\mathrm{N}$ & 362 & 379 \\
\hline
\end{tabular}

Apreciamos diferencias significativas en tratamiento posterior que se hacen de datos generados post AF. De la muestra que afirma que comparten resultados, el 29,7\% ( $\mathrm{N}=52)$ son hombres y el 13,7\% $(\mathrm{N}=28)$ mujeres. Los adolescentes que no comparten sus datos en redes sociales, el 70,3\% $(\mathrm{N}=123)$ son hombres y el $86,3 \%(\mathrm{~N}=176)$ mujeres. Como el pvalor de la prueba de independencia chi cuadrado $(0,000)$ sale significativo $(\mathrm{p}<$ 0,05), podemos aceptar la hipótesis de dependencia entre las variables. Ante esta pregunta podemos apreciar la influencia en las redes sociales, el 90\% afirman haber publicado alguna vez historias o vídeos, realizando AF (Tablas 17 y 18).

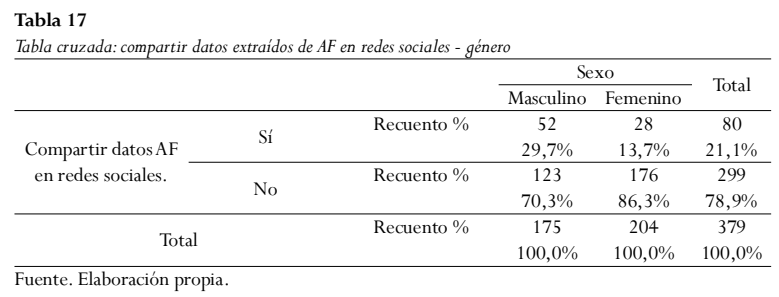

Observamos en la Tabla 18 que la correlación entre estas variables es positiva, ya que el valor obtenido $(0,195)$

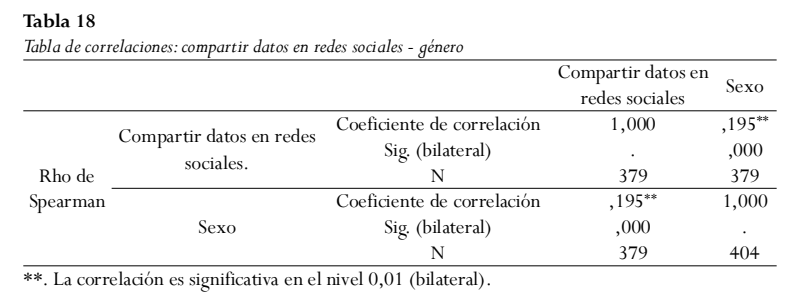

\section{Figura 6}

es superior a -1. Existe una relación proporcional entre el hecho de compartir los datos en redes sociales y el sexo de la población encuestada.

Podemos ver que las personas tratan de proyectar su ocio, que en este caso es la práctica de AF. Las redes se muestran como potenciadoras de hábitos saludables y mejora de la salud a través de su realización donde se intentan viralizar los retos, experiencias de actividad física, prácticas deportivas, etc., (Figura 6).

Observamos la importancia que está teniendo Instagram en los hábitos saludables. La mayoría de la muestra cree que Instagram es un medio que promueve

Post en vuestro perfil en los que se os ve realizando AF. Fuente: elaboración propia.

los hábitos saludables, principalmente al ser una red basada principalmente en la imagen, cuidado del cuerpo, búsqueda de belleza, pero de forma intrínseca adentra también en hábitos saludables. Gran parte de la muestra asocia el cuidado del cuerpo para tener así mejores fotos en Instagram (Figura 7). Por lo tanto, la AF para mantener el cuerpo de una forma más estilizada y la buena alimentación es algo que está influyendo desde las redes.

En público adolescente que comparte AF en redes sociales, como presenta la Tabla 19, con 16 años el 14,2\% publica sus entrenamientos, con 17 años queda representado por un 17,2\%, con 18 años está respaldado por el 33,3\%, el de 19 años representa el 5,3\% y, por último, con 20 años, el 35,5\% afirma compartir dichos datos. Referido a la parte de la muestra que no deciden publicar sus datos, el 85,5\% tienen 16 años, 82,8\% 17 años, 66,7\% 18 años, 94,7\% 19 años y 64,2\% 20 años. El p-valor de la prueba de independencia chi cuadrado $(0,000)$ sale significativo $(\mathrm{p}<0,05)$, por tanto, con un nivel de confianza del $95 \%$. 


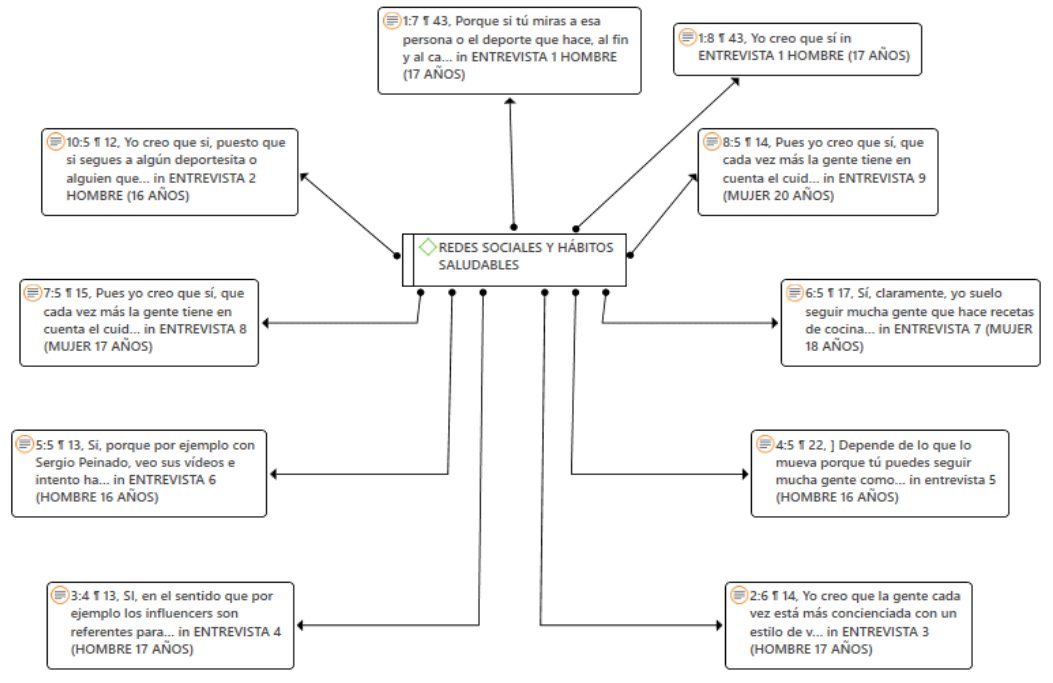

Figura 7

Influencia de Instagram en los hábitos saludables. Fuente: elaboración propia.

consecuencia, sienten admiración por estas personas a las que siguen y confirman que tratan de imitarlos en sus $\mathrm{mov}$ i m i e $\mathrm{n} \mathrm{t}$ o s, influyendo en su AF de forma directa (Simó Quiles, 2018) $\left(\mathrm{H}_{2}\right)$. En función del género del influencer, entre los adolescentes se determina que el masculino tiende a tener un influencer de distinto género,

Tabla 19

Tabla cruzada: compatir datos extraídos de $A F$ en redes sociales-edad

\begin{tabular}{|c|c|c|c|c|c|c|c|c|}
\hline & \multicolumn{5}{|c|}{ Edad } & \multirow[b]{2}{*}{ Total } \\
\hline & & & 16 & 17 & 18 & 19 & 20 & \\
\hline Compartir & & Recuento \% & 29 & 5 & 6 & 1 & 39 & 80 \\
\hline datos extraídos & & & $14,2 \%$ & $17,2 \%$ & $33,3 \%$ & $5,3 \%$ & $35,8 \%$ & $21,1 \%$ \\
\hline $\begin{array}{c}\text { de AF en redes } \\
\text { sociales }\end{array}$ & No & Recuento \% & $\begin{array}{c}175 \\
85,8 \%\end{array}$ & $\begin{array}{c}24 \\
82,8 \%\end{array}$ & $\begin{array}{c}12 \\
66,7 \%\end{array}$ & $\begin{array}{c}18 \\
94,7 \%\end{array}$ & $\begin{array}{c}70 \\
64,2 \%\end{array}$ & $\begin{array}{c}299 \\
78,9 \%\end{array}$ \\
\hline Total & & Recuento \% & 204 & $\begin{array}{c}29 \\
100,0 \%\end{array}$ & $\begin{array}{c}18 \\
100,0 \%\end{array}$ & $\begin{array}{c}19 \\
100,0 \%\end{array}$ & $\begin{array}{c}109 \\
100,0 \%\end{array}$ & $\begin{array}{c}379 \\
100,0 \%\end{array}$ \\
\hline
\end{tabular}

Observamos que la correlación entre estas variables es negativa (Tabla 20), ya que el valor obtenido $(-0,213)$ está por debajo de -1. Interpretamos que el hecho de compartir los datos en redes sociales en relación con edad no es directamente proporcional.

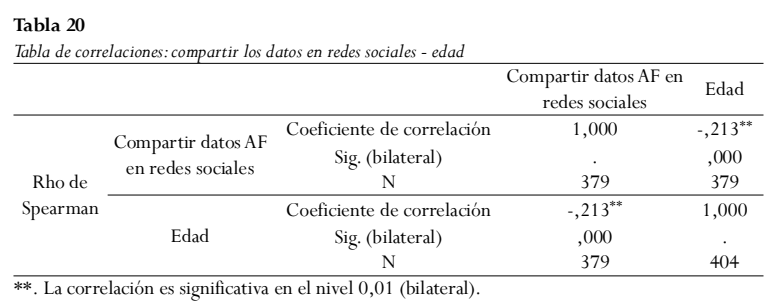

\section{Discusión}

Tener o no tener un influencer deportivo está condicionado por la edad. En nuestro estudio podemos manifestar que las personas con 16 años tienen más probabilidad de tener un influencer deportivo que las personas de 20 años. Sin embargo, en relación con el género, afirmamos que ser hombre o mujer no es determinante para tener o no un influencer deportivo (Serra Puyal, et al., 2018) $\left(\mathrm{O}_{1} ; \mathrm{H}_{1}\right)$. En cambio, la modalidad deportiva practicada influye directamente en la etapa de la adolescencia tardía, independientemente de los años y el género, teniendo especial predilección por seguir, bien a deportistas famosos, o influencers de su modalidad deportiva y, como mientras que el femenino se decanta más por influencer de su igual género, aunque no existe ninguna asociación entre el género del adolescente y el perfil deportivo que se prefiera, ni podemos afirmar que existe correlación entre modalidad deportiva que prefieren ambos géneros, empatando en porcentaje por gustos sobre el fitness, modalidad deportiva que puede, a priori, desinteresar más a género femenino en edades adolescentes (Veiga, et al., 2017) $\left(\mathrm{H}_{3} ; \mathrm{O}_{2}\right.$ y $\left.\mathrm{O}_{3}\right)$.

Las personas en la etapa de la adolescencia tardía que tienen un influencer deportivo sienten más adherencia y motivación por la AF (Greco, et al., 2015). En nuestra investigación se ha observado diferencias significativas a favor de las personas que tienen mayor motivación por la $\mathrm{AF}$, ya que ésta queda supeditada a seguir o no a dichas personas mediáticas $\left(\mathrm{O}_{1}\right)$. Por tanto, las personas que practican AF sienten que sus hábitos deportivos han sido mejorados gracias a la figura del influencer $\left(\mathrm{H}_{4}\right)$ (Aznar et.al, 2019). Los influencers, al igual que la tenencia al uso de dispositivos digitales, aumentan la motivación hacia la práctica diaria de AF.

Centrándonos en el consumo de productos digitales, dicha compra se ve influenciada por el género, destacando el sexo masculino frente al femenino (Estrada-Marcén, et al., 2019), donde las tecnologías fitness son empleadas también por un $68,7 \%$ de los hombres, y un 57,7\% de las mujeres. No podemos determinar para las variables sexo y edad de las personas participantes esta importancia por adquirir un gadget digital porque los resultados no son lo suficientemente dependientes como para establecer diferencias significativas, pues el $\mathrm{p}$ valor para la correlación género es 0,737 y 0,860 para la correlación edad, ambas mayores de $\mathrm{p}>0,05\left(\mathrm{O}_{4}\right)$. No podemos olvidarnos de que las personas que dedican 
más horas a la práctica deportiva han comprado un dispositivo digital (Tironi Rodó, et al., 2018) $\left(\mathrm{H}_{5}\right)$.

En el consumo de productos digitales podemos vislumbrar que la modalidad deportiva practicada puede influir directamente en la adquisición del producto que las personas adolescentes suelen utilizar para correr y para salir con bicicleta, deportes individuales. Por el contrario, las personas que practican deportes colectivos muestran menor interés por la adquisición de estos dispositivos $\left(\mathrm{H}_{6}\right)$. Otro aspecto importante a nivel de marketing es la marca que los influencer patrocinan. En este caso, las personas que han comprado un gadget digital, más de la mitad de los adolescentes, en general, no ha intentado que la marca del dispositivo objeto de compra fuese o igual o parecido al de su influencer (Simón-Grima, et al., 2021) $\left(\mathrm{O}_{4}\right)$.

El hecho de dedicar tiempo a la práctica de AF es un factor influyente para la adquisición de estos aparatos. Más del 70\% de la muestra activa afirma ser propietario de wereables destinados a la AF (Wright, et al., 2018) $\left(\mathrm{H}_{5}\right)$. El estudio refleja que no importa el estrato social al que pertenezcas, el hecho de adquirir un dispositivo no está determinado por ello. Desde el prisma de la actividad en redes sociales (Flores Rivera, et al, 2021, el género influye en que se compartan datos en las redes sociales, siendo los hombres quienes muestran más interés en esta práctica. La edad también influye en que se compartan o no los datos en las redes sociales (H7) $\left(\mathrm{O}_{5}\right)$. Los sujetos de 20 años prefieren compartirlos con un $48,8 \%$, frente al extremo representado por la población de 16 años, la cual no comparte los datos con un 58,8\% de afirmación (Roelands \& Pauw, 2019) $\left(\mathrm{H}_{7}\right)$ $\left(\mathrm{O}_{5}\right)$.

\section{Conclusiones}

El mundo de los influencers deportivos queda demostrado que ejerce influencia sobre el consumo, la práctica de la AF y la proyección que hacen los prosumidores andaluces (España) en las redes sociales. Esta interacción va aumentando progresivamente y se encuentra determinado por factores como la edad, el género y el nivel de AF que se realiza. Este estudio quiere ser una aportación más a investigaciones más amplias con el fin de concretar aún más el campo de acción que la realidad de los influencers ha abierto en la sociedad Postdigital.

Una vez más se hace imprescindible que los agentes educativos y sociales, como influencers de aprendizaje, apuesten por la alfabetización mediática de las personas que pasan por la etapa de la adolescencia. Para 2030 la ONU nos ha lanzado el reto de trabajar para el logro de los objetivos del desarrollo sostenible; el objetivo 4, de una educación de calidad, nos presenta esta demanda clave como es la alfabetización de toda la ciudadanía en todos los niveles. Apostemos por el pensamiento crítico, asentemos una actitud crítica y, como consecuencia, tendremos una sociedad democrática.

\section{Referencias}

Anuario de Estadísticas Deportivas (2019). División de Estadística y Estudios. Ministerio de Cultura y Deporte. Recuperado de: https://cutt.ly/gQqHC7w

Aznar, I., Romero, J.M., \& Rodríguez-García, A.M. (2018). La tecnología móvil de Realidad Virtual en educación: una revisión del estado de la literatura científica en España. EDMETIC, Revista de Educación Mediática y TIC, 7(1), 256-274. http://doi.org/ 10.21071/edmetic.v7i1.10139.

Corbetta, P. (2007). Metodología y técnicas de investigación social. McGawHill.

Encuesta Nacional de Salud (2012). Instituto Nacional de Estadística. Ministerio de Sanidad, Servicios Sociales e Igualdad. Recuperado de: https: / / cutt.ly/ LvcQLjX

Encuesta Europea de Salud (2014). Encuesta Europea de Salud en España. Recuperado de: https: / / cutt.ly/ $\operatorname{lm} 3 \mathrm{HTMz}$

Escaño, C. (2019). Sociedad Postdigital (ontología de la remezcla). https://cutt.ly/WrMr37f

Estrada-Marcén, N., Sanz-Gonzalo, G., Simón-Grima, J., Casterad-Seral, J., \& Roso-Moliner, A. (2019). Los centros de fitness de la ciudad de Zaragoza.Apunts: Educación Física y Deportes, 4(135), 118-136. http:/ / doi.org/doi.org/10.5672/apunts. 2014 0983.es.(2019/1).135.08.

Flores Rivera, C., Luna Villouta, P., Fuentealba Urra, S., Garrido Méndez, A., Muñoz Sabando, G. \& Torres Esparza,A. (2021). Significados atribuidos a la práctica de actividad física, ejercicio físico y deporte como medio de configuración de redes sociales y participación ciudadana. Retos, 42, 831-840. https: / /doi.org/10.47197/retos.v42i0.65967

García, M., Alvira, F., Alonso, L., \& Escobar, M. (2015). El análisis de la realidad social. Métodos y técnicas de investigación. Alianza Editorial.

García-Fernández, J., Fernández-Gavira, J., DuránMuñoz, J., \& Vélez-Colón, L. (2015). La actividad en las redes sociales: Un estudio de caso en la indus- 
tria del fitness. Retos, 28, 44-49. https://doi.org/ $10.47197 /$ retos.v0i28.34839 Romero-Rodríguez, L.M. (2020). Learning Leaders: Teachers or Youtubers? Participatory Culture and STEM Competencies in Italian Secondary School Students. Sustainability 12, 7466. https://doi.org/ $10.3390 /$ su12187466

Gil-Quintana, J., Santoveña-Casal, S., y Romero Riaño, E. (2021) Realfooders Influencers on Instagram: From Followers to Consumers. Int. J. Environ. Res. Public Health, 18, 1624. https://doi.org/10.3390/ ijerph18041624

Greco, G., Tambolini, R., Ambruosi, P., \& Fischetti, F. (2017). Negative effects of smartphone use on physical and technical performance of young footballers. Journal of Physical Education and Sport. 17(4), 2495-2501. http://doi.org/10.7752/ jpes.2017.04280.

Informe Anual 2019. La Sociedad en Red. Transformación digital en España. Ministerio de Economía y Empresa. Recuperado de: https://cutt.ly/2m3H5Vj

Jensen, M. M. \& Mueller, F. (2021). Running with technology: Where are we heading? 26th Australian Computer-Human Interaction Conference, OzCHI 2014. Sydney, New South Wales, Australia. http: / / doi.org/10.1145/2686612.2686696.

Martínez Lemos, I. \& González-Sastre, B. (2016). El mercado del fitness en España: Un estudio exploratorio sobre el perfil económico del segmento low-cost. Cultura, Ciencia y Deporte, 11, 33. http: / /doi.org/10.12800/ccd.v11i33.764.

Moreno Muciño, O., Medina Rodríguez, R., Enríquez Reyna, M., González, J., \& Ceballos Gurrola, O. (2021). Actividad física y uso de redes sociales en estudiantes de secundaria. Diferencias por sexo y grado escolar. Retos, 42, 276-285. https://doi.org/
Gil-Quintana, J., Malvasi, V., Castillo-Abdul, B., y

\subsection{7/retos.v42i0.86364}

Ricoy, C. (2006). Contribución sobre los paradigmas de investigación. Revista do Centro de Educação, 31 (1), 11-22. http: / / doi.org/10.5902/198464441486.

Roelands \& Pauw, D. (2019). Technological Impact on Human Performance. International Journal of Sports Physiology And Performance. http: / / doi.org/10.1123/ ijspp.2018-0941.

Tironi Rodó, M., \& Valderrama Barragán, M. (2018). Transformando la bicicleta en laboratorios móviles. Antropología experimental, 16. https://doi.org/ 10.17561 /rae.v0i16.3016

Simó Quiles, A. (2018). Investigación del papel de dos deportistas famosos, como influencers, en la red social Instagram. Recuperado de: http: / / hdl.handle.net/10251/114863

Simón-Grima, J., Estrada-Marcén, N., \& Serrano Ostáriz, E. (2019). Tecnología digital para la salud y la actividad física (pp. 147-165). Madrid, España: Ediciones Pirámide.

Serra Puyal, J.R.; Zaragoza Casterad, J., \& Generelo Lanaspa, E. (2014). Influencias de «otros significativos» para la práctica de actividad física en adolescentes. Revista internacional de Medicina y Ciencias de la Actividad Física del Deporte 14.56 (2014): 735-753 Recuperado de: https:/ / cutt.ly/LvvDGf9

Veiga, Ó. L., Valcarce Torrente, M., \& King-Clavero, A. (2017). Encuesta nacional de tendencias de fitness en España para 2017. Apunts. Educacion Fisica y Deportes, 128, 108-125. http://doi.org/10.5672/ apunts.2014-0983.es.(2017/2).128.07.

Wright, S. P., Hall Brown, T. S., Collier, S. R., \& Sandberg, K. (2017). How consumer physical activity monitors could transform human physiology research. American Journal of Physiology. 312(3), R358R 367 .http: / / d oi .org/ 10.1152 / ajpregu.00349.2016.
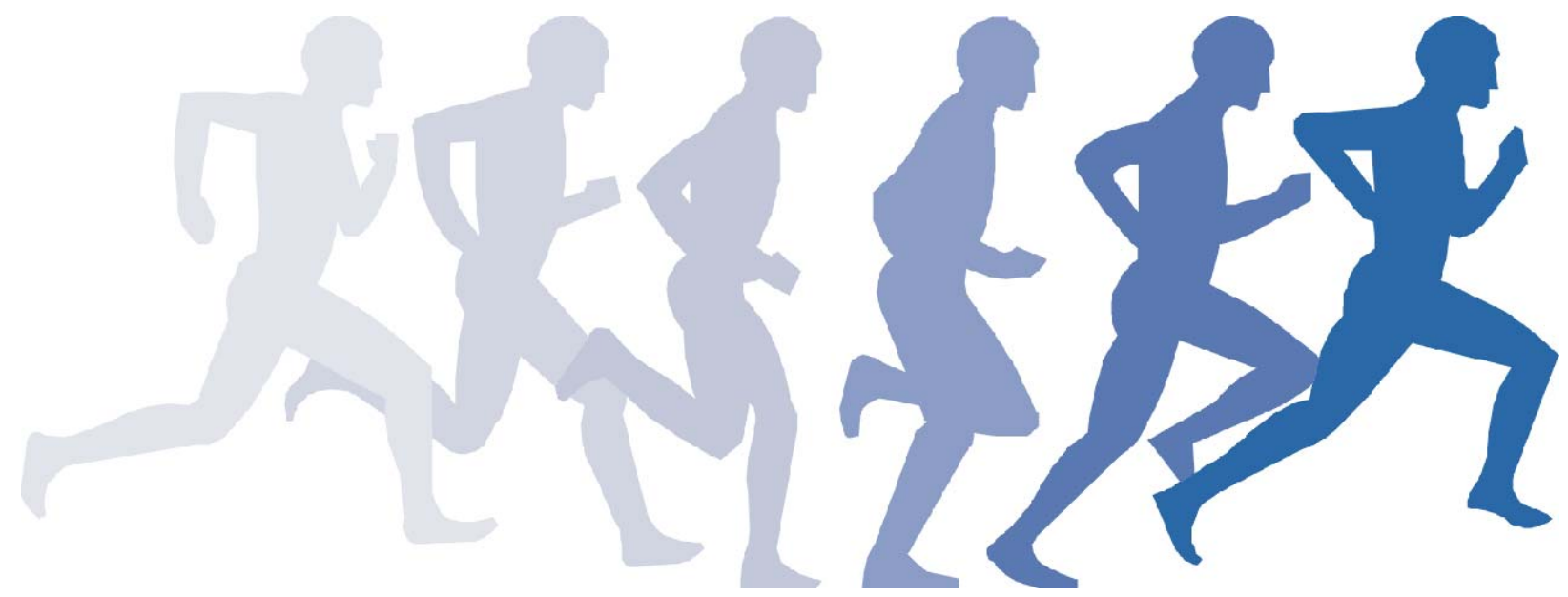